\title{
Principles, Anatomical Origin and Applications of Brainwaves: A Review, Our Experience and Hypothesis Related to Microgravity and the Question on Soul
}

\author{
Zamzuri Idris1,2, Mustapha Muzaimi ${ }^{1,2}$, Rahman Izaini Ghani1,2, Badrisyah Idris ${ }^{1,2}$, \\ Regunath Kandasamy ${ }^{1,2}$, Jafri M. Abdullah ${ }^{1,2}$ \\ ${ }^{1}$ Center for Neuroscience Service and Research, Universiti Sains Malaysia, Kubang Kerian, \\ Malaysia \\ ${ }^{2}$ Department of Neurosciences, School of Medical Sciences, Universiti Sains Malaysia, Kubang Kerian, \\ Malaysia \\ Email: neuroscienceszamzuri@yahoo.com
}

Received 6 April 2014; revised 20 May 2014; accepted 3 June 2014

Copyright (C) 2014 by authors and Scientific Research Publishing Inc.

This work is licensed under the Creative Commons Attribution International License (CC BY). http://creativecommons.org/licenses/by/4.0/

(c) (i) Open Access

\section{Abstract}

Brainwave is a kind of traceable neurophysiological energy in a living brain. Invisible to human eyes, it is only detectable using electroencephalography (EEG), electrocorticography (ECoG) and magnetoencephalography (MEG). The waves or oscillations or rhythms are produced mainly by the oscillatory networks of the brain. Three main oscillatory networks are thalamocortical, extrathalamic-cortical and cortical-cortical networks. Greater limbic system (reticular system, hypothalamus, thalamus, basal forebrain nuclei, limbic system) has a great influence on these oscillatory networks. This system which is in microgravity position lies deep inside and surrounded by the ventricles of the brain. It receives all information from inside and outside of our body and then projects to all areas of the brain (from all to one and from one to all-nearly similar concept to "from God back to God"). Therefore, the greater limbic system could be regarded as "a core of the neuroaxis" which lies in microgravity compartment and in microgravity position (" $T$ "-shape or curving shape; whilst gravity position is " $\mathrm{I}$ "-shape or vertical shape). By knowing the origin of the brainwaves and methods to detect them, one may study seizure networks, normal and abnormal brain networks and arguably, even to explore the relationship between the "invisibles": "invisible" brainwaves and "invisible" soul.

\section{Keywords}

Brainwaves, Electroencephalography, Electrocorticography, Magnetoencephalography, Reticular

How to cite this paper: Idris, Z., et al. (2014) Principles, Anatomical Origin and Applications of Brainwaves: A Review, Our Experience and Hypothesis Related to Microgravity and the Question on Soul. J. Biomedical Science and Engineering, 7, 435-445. http://dx.doi.org/10.4236/jbise.2014.78046 


\section{Introduction}

Oscillation with synchronisation does exist inside and outside of our brain. Neural oscillation can be stratified into microscale-oscillation (activity of a single neuron), mesoscale-oscillation (activity of local group of neurons or vertices) and macroscale-oscillation (neural activity of different brain regions/networks) [1]. Neurons can generate action potentials or spike trains (multiple action potentials in sequence) at microscale oscillation and can be studied using intracellular single-unit recordings. When a group of neurons fire action potentials, synaptic interactions play a major role to synchronise the input to other brain regions. Synchronised firing patterns give rise to large-amplitude mesoscale oscillations of local field potentials which can be detected as brainwaves by using electroencephalography (EEG), electrocorticography (ECoG) or magnetoencephalography (MEG). Neural oscillations which arise from interactions between or among brain regions are known as macroscale-oscillation and form various brainwaves network loops or circuits inside our brain [2]-[5]. This article describes the principles for the brainwaves, their anatomical origin and relationship with microgravity, and our experience in using them to study brain and seizure networks, mapping the region of the brain and lately to explore the relationship between body, mind and soul.

\section{Principles of Brainwaves}

Brainwaves can be detected using EEG, ECoG and MEG. There are five major brainwaves distinguished by their different frequency ranges from high to low frequency: a) gamma (above $30 \mathrm{~Hz})$; b) beta (13 - $30 \mathrm{~Hz})$; c) alpha (8 - $13 \mathrm{~Hz})$; d) theta $(4-8 \mathrm{~Hz})$ and e) delta $(0.5-4 \mathrm{~Hz})$ [6]. Slow waves are waves frequencies of less than $8 \mathrm{~Hz}$, including theta and delta waves whilst fast waves are waves of more than $13 \mathrm{~Hz}$. Hertz is a unit of frequency which equals cycle per second (Hz: cycle/sec). Amplitude of brainwaves is normally measured using microvolt unit $(\mu \mathrm{V})$. Low amplitude falls within less than $20 \mu \mathrm{V}$, medium amplitude between $20-50 \mu \mathrm{V}$ and high amplitude, more than $50 \mu \mathrm{V}$. Pertaining the types of brainwaves, alpha waves (8 - $13 \mathrm{~Hz} ; 20-60 \mu \mathrm{V})$ mainly appear in the posterior half of the head and are usually found over the parieto-occipital region of the brain. It may appear as round, sinusoidal or sometimes as sharp waves and regarded as the most prominent rhythm in brain activity. It normally indicates a relaxed awareness without any attention or concentration [7]. Concerning beta wave (13 - 30 Hz; $2-20 \mu \mathrm{V})$, it is the usual waking rhythm of the brain associated with active thinking or active attention (fast wave). In adults, it is normally found at frontal and central region [7]. The slow wave theta $(4-8 \mathrm{~Hz} ; 20-100 \mu \mathrm{V})$ is thought to originate from the thalamus. It appears as consciousness slips towards drowsiness. Theta waves therefore have been associated with sleep, deep meditation, creative inspiration and unconsciousness [6]-[8]. Another slow wave is delta wave $(0.5-4 \mathrm{~Hz} ; 20-200 \mu \mathrm{V})$. It is associated with deep sleep and unconsciousness [7] [8]. Both slow waves can play normal role in newborn, infancy and childhood [9]. Lastly, the gamma wave (above $30 \mathrm{~Hz}$; approximately $5-10 \mu \mathrm{V}$ ) which were reported by Galambos et al. in 1981 occurs at certain occasion when sensory stimuli such as auditory clicks or flashes of light were given [10]. Recently, it was reported as a good indicator of event-related synchronisation of the brain and therefore can be used to demonstrate the locus for the activity [11] [12]. Other types of brainwaves are: a) Kappa waves_-found at $10 \mathrm{~Hz}$ and associated with thinking; b) Lambda waves_occurring at occipital region which associated with visual exploration; c) Mu waves-recorded at Rolandic fissure (vertex of scalp) in alpha frequency ( $8-13 \mathrm{~Hz}$ ) but it is independent of alpha, blocking not with eye opening but with movement or intended movement; d) Rho waves - are positive transient sharp waves at occipital region; and e) Spindle (sigma) waves - waxing and waning spindle waves at $7-14 \mathrm{~Hz}$, that indicates EEG synchronisation recorded during quiescent sleep [6] [7] [9].

\subsection{EEG and ECoG}

Surface or scalp EEG has low signal resolution owing to the presence of scalp, bone and dura covering the brain. This greatly reduces the amplitude of the brainwaves. Higher signal resolution for scalp EEG can be achieved by modifying the number of the electrode, the electrode contact and sensor, recording system such as the amplifier 
and filter, and wireless system. The future EEG should make the channel numbers higher in order to nearly sample all areas of the brain. Currently, the EEG channels are commonly available in 16, 32, 64, 128 channels, but lately the EEG with 256 channels are available (EEG-1200 Neurofax, Nihon Kohden, Japan) and Dense array EEG, Geodesic Sensor Net (EGI, Inc, Eugene OR, USA). Dry electrode contact and wireless system are already in the market (Mindo, Contec etc.) [13] [14]. Certainly, improvement in EEG sensor and recording technology offers a wider and improved prospect for its application. The nanotechnology may change many aspects of the EEG, for example for "each" electrode sensor, it has been incorporated with a nanoamplifier system [15]. The use of miniaturized and more sensitive nanomaterials for sensors, the higher the number of electrode-sensors and signal detection can be made. Together with the advancement in signal processing, the short-lived brain signals can be made possible via scalp EEG. In short, the 10 - 20 system is commonly used to allocate the scalp electrodes. 10 - 20 denotes various electrode locations are either $10 \%$ or $20 \%$ of the distance between standard points used for measurement. In relation to the recording technique, one may use either monopolar or bipolar technique. A monopolar technique involves placing one active electrode in area of interest and another electrode termed reference electrode on a relatively inactive area such as earlobe, or tip of the nose. This technique is convenient where activity produced at a specific area is monitored and in recording event-related potentials (ERP). On the contrary, the bipolar recording technique uses two active electrodes placed over cortical areas of interest. It records the difference of electrical potentials between two regions at any instant. However, combined recorded activities at two locations is an apparent disadvantage and therefore interpretation should be made by comparing the symmetrically placed electrodes [right with left (or vice versa) hemisphere] [6]. In addition, EEG is employed in a combination technique, most commonly involving EEG-fMRI and EEG-MEG. Such combinations improve the localisation of the abnormal area and widened the scopes of research in the related areas. ECoG or intraoperative EEG (iEEG) uses the same principles as the EEG; it differs in term of the actual site of the recording - onto the surface of brain cortex (used grid or strip electrodes) or deep inside the brain (recorded using depth electrodes). Therefore, it provides brain signals that have an exceptionally high signal-to-noise ratio, less susceptibility to artefacts than current scalp EEG, and have a high spatial and temporal resolution $(<1 \mathrm{~cm} /<1$ millisecond respectively). Since it is an invasive procedure, it is less often used with exceptions in brain mapping during awake brain surgery and in epilepsy surgery [16]-[18].

\subsection{MEG}

MEG is non-invasive (Figure 1(a)). It is similar to EEG but is based on magnetic field changes (rather than voltage/potential changes as in EEG) that accompany neuronal activity. Magnetic evoked and spontaneous fields are recorded while patients seated in a magnetically shielded room (MaxShieldTM, Elekta Oy, Helsinki, Finland) using a 306-channel (102 magnetometers and 204 gradiometers) whole-head MEG system (Elekta Neuromag ${ }^{\circledR}$, Elekta Oy, Helsinki, Finland). A third-order software gradient is used after online band-pass filtering between $0.1-300 \mathrm{~Hz}$ to discard noises. The sampling frequency is typically $1 \mathrm{KHz}$. The head position relative to the MEG sensors of the helmet is localized using: a) 3 fiducial localization coils attached to right and left preauricular points, and the nasion of the patients; b) 100 - 150 points digitized around the head using a 3D positions monitoring systems (Pholemus, Colchester, VT); c) four electromagnetic head positions indicator (HPI) coils are used to assess the head position at the beginning of the measurement process. During recording, the head-position changes of up to $1.5 \mathrm{~cm}$ are acceptable. The anatomical brain images (T1/2/FLAIR/3D) are also obtained using MRI. Fusion between the anatomical MRI images and tomographic reconstruction of the head-model brainwave data is usually done prior to source localization and this is called magnetic source imaging (MSI) [19]. There are two main types of MEG recording for brainwaves: a) Evoked or event related fields. Evoked or event activity is the brain responses that are directly related to stimulus-related activity. Evoked fields are commonly used for motor, sensory, visual and auditory mapping; whilst event related fields are mainly used for cognitive tasks; b) resting state activity or spontaneous activity are used mainly in detecting seizure focus, studying brain maturity, consciousness and brain networks. MEG source localizations for somatosensory, motor, auditory and visual evoked magnetic fields were performed normally by using the technique of overdertermined equivalent current dipoles (ECD). Complex analysis using underdetermined (modelling) technique is used for source localisation in cognitive or event related field, whilst collection of events which based on ECD technique is used to study seizure and brain networks [1]. 


\subsection{Oscillations and Waves}

Interestingly, the recorded shapes of sinusoidal brainwaves have two main features: up (or down) and down (or up) (i.e. in oscillation) nearly similar to action potentials waves. This up (down) and down (up) shape (waves) is also seen in other body systems such as in the lungs during respiration, in the cardiovascular system during heart beating, in the vascular system which known as Windkessel phenomenon or effect causing macrocirculatory pulsation, gastrointestinal system during digestion and gustatory movements and even at the atomic level, the oscillating atoms producing wave-like features. When viewed outside of our body and from the time of Big Bang (theory for the creation of the universes) until now, all matters are also in oscillations including the light waves. Therefore, understanding oscillatory activity (cycle/sec) seems crucial for us in order to pinpoint the origin and study the functions of brainwaves. The anatomical study done by Moruzzi and Magoun in 1949 revealed reticulo-thalamic and reticulo-extrathalamic projections may play important roles in generating brain oscillations or waves [20] [21].

\section{Origin of Brainwaves: Greater Limbic System (? Seat of the Soul) and Microgravity}

The origins of brainwaves or EEG/MEG/ECoG detected brainwaves are mainly from pyramidal postsynaptic potentials which are in oscillations with: a) the thalamus, therefore known as thalamocortical networks (modulated by the reticulo-thalamo-cortical circuits. It is part of greater limbic system: dorsal pathway); b) the extrathalamic-cortical circuits (mainly involve the reticular system, hypothalamus, hippocampus, amygdala, basal forebrain nuclei - they are part of the greater limbic system too: ventral pathway) and c) other cortex, known as cortical-cortical networks [6] [20]-[23]. Moruzzi and Magoun in 1949 had shown the involvement of the reticular system inside the brainstem for its crucial role in generating pattern of brainwaves [20] [21]. This anatomical region (classical reticular system inside the brainstem) has vast networks with other structures in the diencephalon (thalamus, hypothalamus, basal forebrain nuclei, parahypothalamic nuclei, pineal and pituitary glands, limbic system) and even with the telencephalon (insula, basal ganglia and neocortex) [24]. It is also the centre regarded by most to be responsible for consciousness, sleep and dreams and in integrating behavior, endocrine, autonomic and somatomotor systems [6] [24]. Since it has vast connections with hypothalamus, thalamus, parahypothalamic nuclei, basal ganglia, spinal cord, cerebellum, limbic structures, neocortex (all areas), Nieuwenhuys and colleagues have expanded the definition of classical limbic system caudally and named "greater limbic system" which includes also this classical reticular system which lies inside the brainstem [8] [24] [25]. The greater limbic system forms cores and paracores for the neuroaxis which is in microgravity (Figure 2(a): curving/flexure/bowing/sujood/antigravity/astronaut like) position and generally can be divided into 2: a) reticular activating system (RAS) and b) reticular inhibitory system (RIS) (25). They are extraordinarily rich in neuromediators, such as: a) locus coeruleus (noradrenergic); b) raphe nuclei (serotoninergic/5-HT); c) reticular junction (cholinergic), tuberomammilary nucleus (histaminergic) and d) gamma-aminobutyric acid for reticular inhibitory system (GABA). The dendrites of the cells in this area are arranged in mosaic. In summary, this greater limbic system forms a centre or core for the neuroaxis (? seat of the soul). All information from inside (self) and outside of our body (i.e. universes) goes to this region and from here it projects out to all areas of the brain (all to one and one to all concept). One of the proofs for this area getting involved in generating brain rhythms is the effectiveness of left vagal nerve stimulation in treating refractory epilepsy. The afferent fibres of the vagal nerve have broad projections to the reticulo-hypothalamic-centromedian thalamic-limbic systems or better known as "greater limbic system" via the nucleus of the solitary tract and locus coeruleus [26].

\section{Greater Limbic System and Microgravity}

When further anatomical observation is made at this region, its shape is in "T" shape. The diencephalon and telencephalon are in horizontal position whilst the brainstem is in vertical position. This feature is mainly because of the presence of main cephalic fissure during central nervous system (CNS) development [24]. This curving or flexure position looks like similar to the astronaut-position while in the outer space or in microgravity chamber. The microgravity position (T-shape) of this region is believed to result from the presence of buoyancy inside the CNS. The buoyancy or weightlessness is provided by the CSF and maintained by the anchoring effects of the spinal cord and brainstem nerve roots, ligaments, and by the vascular pulsation provided via Wind- 
kessel effect. This position is similar to the fetus at early gestation while in the uterus (during CNS development) and also similar to all the animals (from invertebrates to vertebrates animals) either from ocean (the largest buoyancy environment on earth) or on land (they are in flexure position or microgravity or horizontal position; but humans are in vertical or "I" gravity position). Considering this Archimedes concept, the inclusion of reticular system into the greater limbic system as advocated by Nieuwenhuys et al. seems valid mainly because, when microgravity (weightlessness) is eliminated, the anatomy of this region is actually form one single vertical connecting core or in gravity position which could be the centre or the seat of the soul [Figure 2(b)—Gravity (weight) position)].
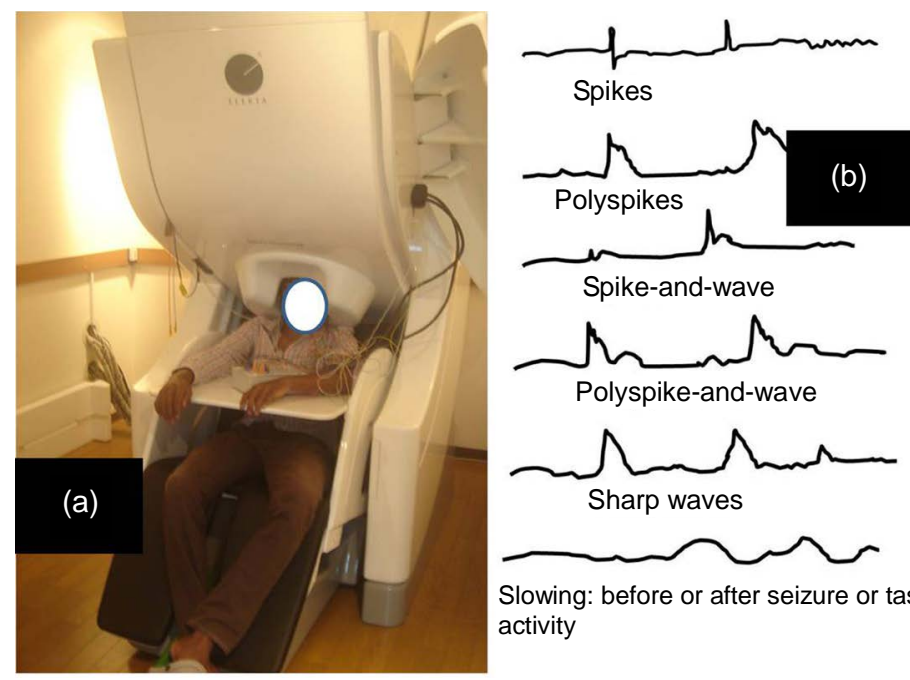

Slowing: before or after seizure or task activity

Figure 1. (a) The magnetoencephalography examination. (b) Brainwaves pattern in detecting seizure.

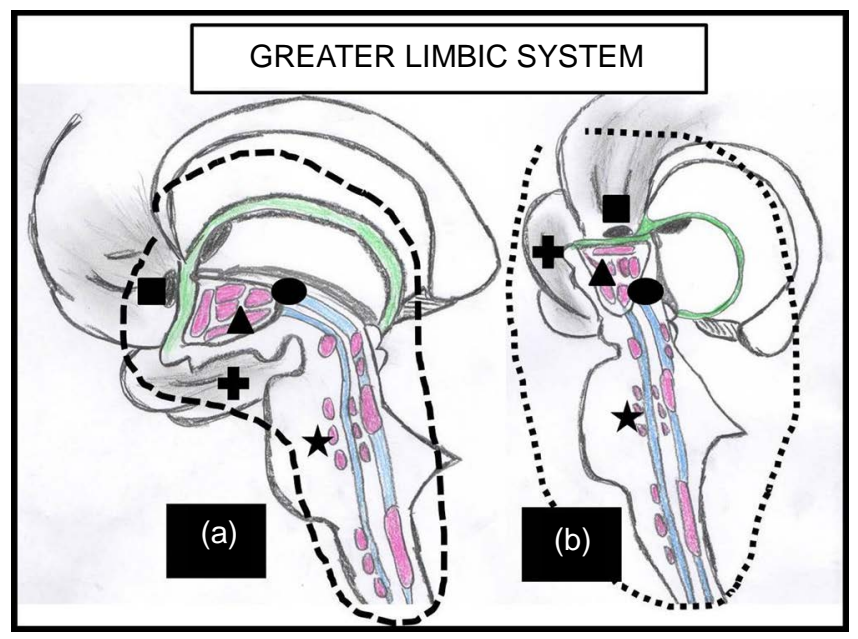

Figure 2. The effect of the microgravity onto the reticular system and brainstem-diencephalic region. (a) Microgravity position and (b) The gravity position. The limbic-hypothalamic-parahypothalamic system extends caudally to the brainstem reticular system when viewed especially in gravity position (which corresponds to Nieuwenhuys and colleagues' idea of greater limbic system). Annotations: squared black-basal forebrain nuclei area; triangle-the hypothalamus; round - the thalamus; star-the mesopontine nuclei/reticular system (locus coeruleus, pedunculopontine nucleus, raphe nucleus); and crossed-the amygdala and hippocampus located lateral to the midbrain area. 


\section{Application of Brainwaves in Our Centre-Example of Usage in Patient (Body) and Research (Soul)}

In our centre, brainwaves were recorded for routine and research purposes. Currently at our centre, brainwaves were recorded using scalp EEG, MEG, ECoG and EEG-fMRI. This is mainly done for epilepsy, brain mapping, and study on brain networks and soul searching. Examples are given below.

\subsection{Epilepsy (Seizure Disorders) and Brain Mapping}

Epilepsy is a seizure disorder that occurs when there is a sudden, brief change in how the brain works and commonly manifested in form of alteration in consciousness, abnormal movements or sensations. Brainwaves features can be slowing waves, spikes, spike-and-wave, polyspikes, polyspike-and-wave and sharp waves (Figure 1(b)). Figure 3(a) shows MEG recordings from a patient suffered from insular epilepsy. She had poor memory, frequent sudden attacks of unable to breath and imbalance. The abnormal brainwaves noted were slowing and sharp waves. The MEG source localization using ECD technique localizes the abnormal waves at periatrial area on both sides which correspond to the lesions inside the ventricles (Figure 3(b)). Mapping this area with the atlas revealed the dipoles for abnormal waves are adjacent to the insular and limbic system (Figure 3(c)). Another refractory epilepsy patient, the epileptic foci were detected using ECoG (Figure 4(a)) and MEG (Figure 4(b)). The patient suffered from focal seizure which involved his right upper limb. The MSI localized the epileptic spikes at the left sensorimotor cortex (Figure 4(c)) which tallied with the intraoperative EEG (iEEE or ECoG) findings (Figure 4(d)). The nearby lesion was removed and eloquent areas with spikes were treated with multiple subpial transections.

\subsection{Brain Networks (Networks for the Pain)}

An army trainee suffers from complex regional pain syndrome (CRPS) after being hit at the left shoulder. His symptoms persist despite various therapies given. His spontaneous resting state MEG with eyes open disclosed abnormal brainwaves or rhythms (Figure 5(a)). Neuromag collection of events software was utilised to analyse the spontaneous brainwave data (brainwave threshold-amplitude was set at above 10\% from normal brainwave activity for brain networks analysis involving high amplitude and abnormal brainwaves morphology-Figure 5(a)). Figure 5 shows the brainwaves collections of events which were localized to the MRI (known as magnetic source imaging or MSI). Multiple activated dipole areas were noted: a) sensorimotor cortices; b) insula cortices (operculum); c) cingulate and d) amygdalo-piriform-hippocampal area (Figure 5(b), Figure 5(c) and Figure 5(d)). These areas corresponded to the pain networks or areas identified in various studies [27]-[30].

\subsection{Brainwaves and the Soul (Spirit)}

Soul or spirit cannot be seen by our eyes. Various texts and manuscripts had described the concept of "behind a living body there is likely a soul”. This soul is commonly related to: a) breathing; b) heart; c) lungs; d) brain; e) immortal; f) from God; g) at the anterior portion of the ventricle as described by Leonardo da Vinci; h) related to CSF containing ventricles; i) knowing the soul, knowing the God better; j) non-substance soul; k) material for the body and immaterial for the soul and last; l) soul is related to an infinite spirit [31] [32]. Following deaths, the physical bodies can no longer function. Those that cannot be noted anymore after death (most are oscillatory items) we can regard as "soul indirect manifestations". Those (soul manifestations) are heart rate, blood pressure, peristalsis, vascular pulsation, breathing movements and "brainwaves". Manifestation of soul in the various forms can easily be understood as an analogy to the light (as soul) and the rainbow (as items in oscillations). Rainbow is the one that can be noted by us, but not the bright light.

During ECoG mapping of the brain under awake state for the seizure spikes at right sensorimotor cortex and at the same time patient was given the option to listen to religious text of the patient's faith, the seizure spikes and the brainwaves were noted to have changed in their morphology (Figure 6(a) and Figure 6(b)). The finding is interesting because it may suggest that the greater limbic system (origin of the brainwaves) is also the spiritual area of the brain (? seat of the soul-where all signals arising from human activities are concentrated/modulated/ processed here). Similar observation on another patient after removal of the lesion at left auditory (dominant hemispheric) area appeared to again show brainwave changes during listening to the religious text (Figure 6(c) and Figure 6(d)). 


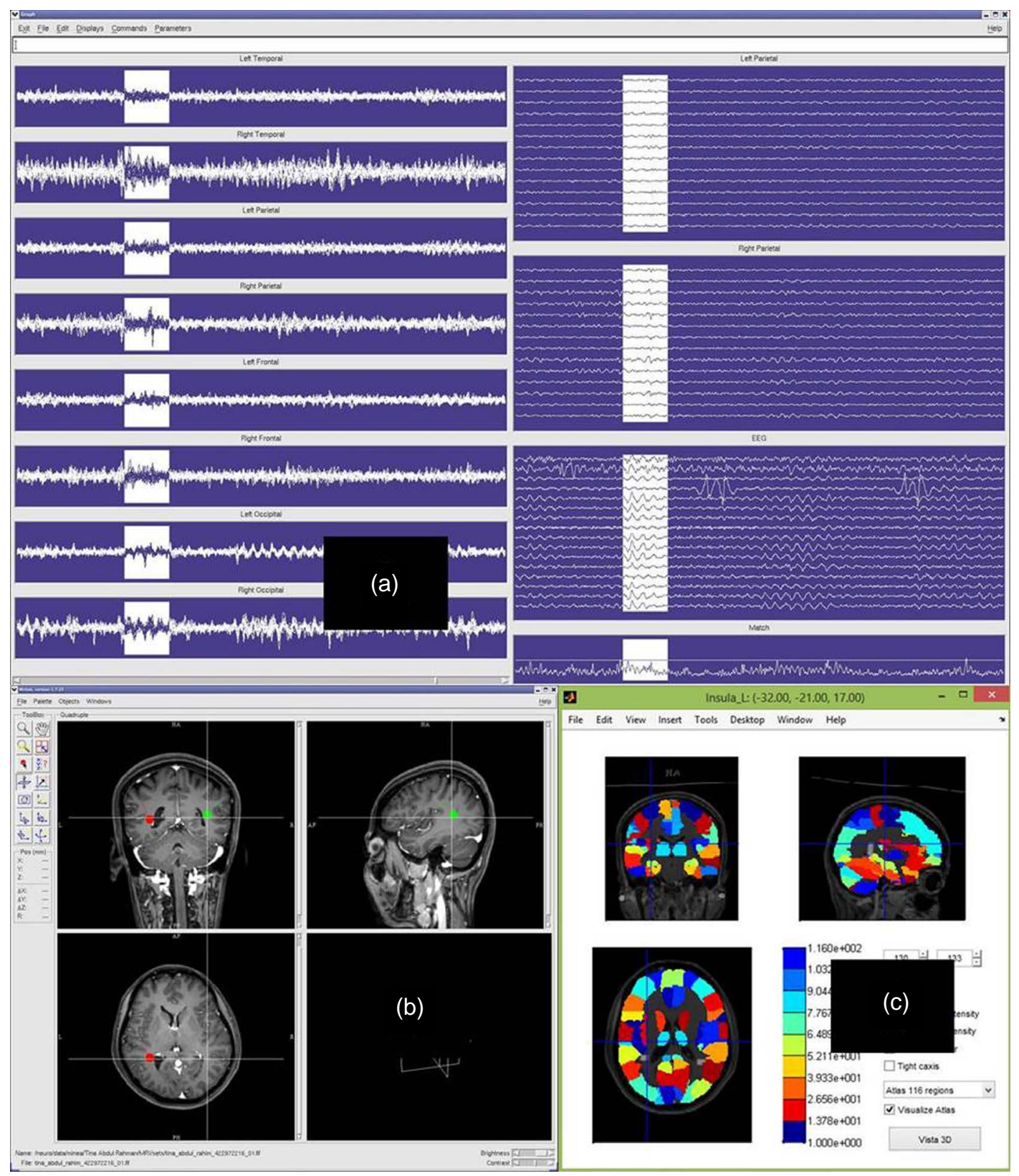

Figure 3. (a) Brainwaves for patient suffering from insular epilepsy. (b) The magnetic source imaging localizes the abnormal waves using equivalent current dipole technique (red and green dipoles). (c) The brain atlas fused to the brainwaves data to know exactly the location for abnormal waves (left insular).

\section{Conclusion}

Greater limbic system and cortical-cortical networks seem crucial in generating brainwaves. These systems cover the whole part of the brain and therefore are important in inducing various functions of the brain via "oscillations" or brain rhythms. Therefore, brainwaves (sinusoidal oscillations) can be further classified based on their frequency. At our centre, normal and abnormal frequencies were studied in many subjects including epilepsy, brain mapping, brain networks and even in the pursuit of "soul searching".

\section{Acknowledgements}

Many thanks to the staffs at our centre in MEG, EEG-fMRI, EEG-ECoG labs; especially Mr Wan M. Affendi, 


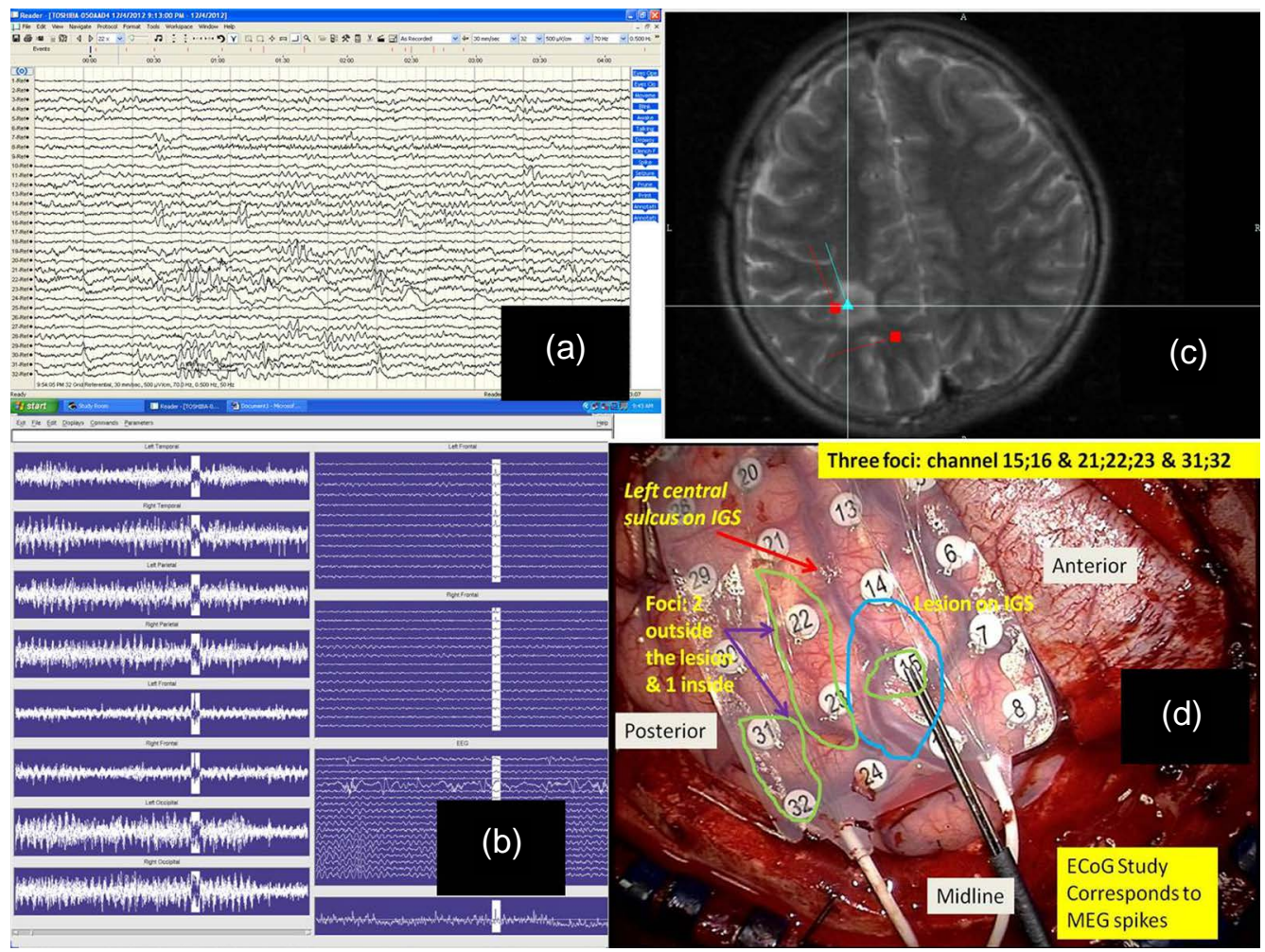

Figure 4. Brainwaves with the seizure spikes detected using ECoG (a) and MEG (b). (c) Source localization identifies the seizure foci near the area of brain lesion at left motor cortex (red squares and blue triangle). (d) Intraoperative EEG localization (iEEE or ECoG) of seizure foci (base on electrode numbers-in A image) correspond with the MEG findings (drawn in green).

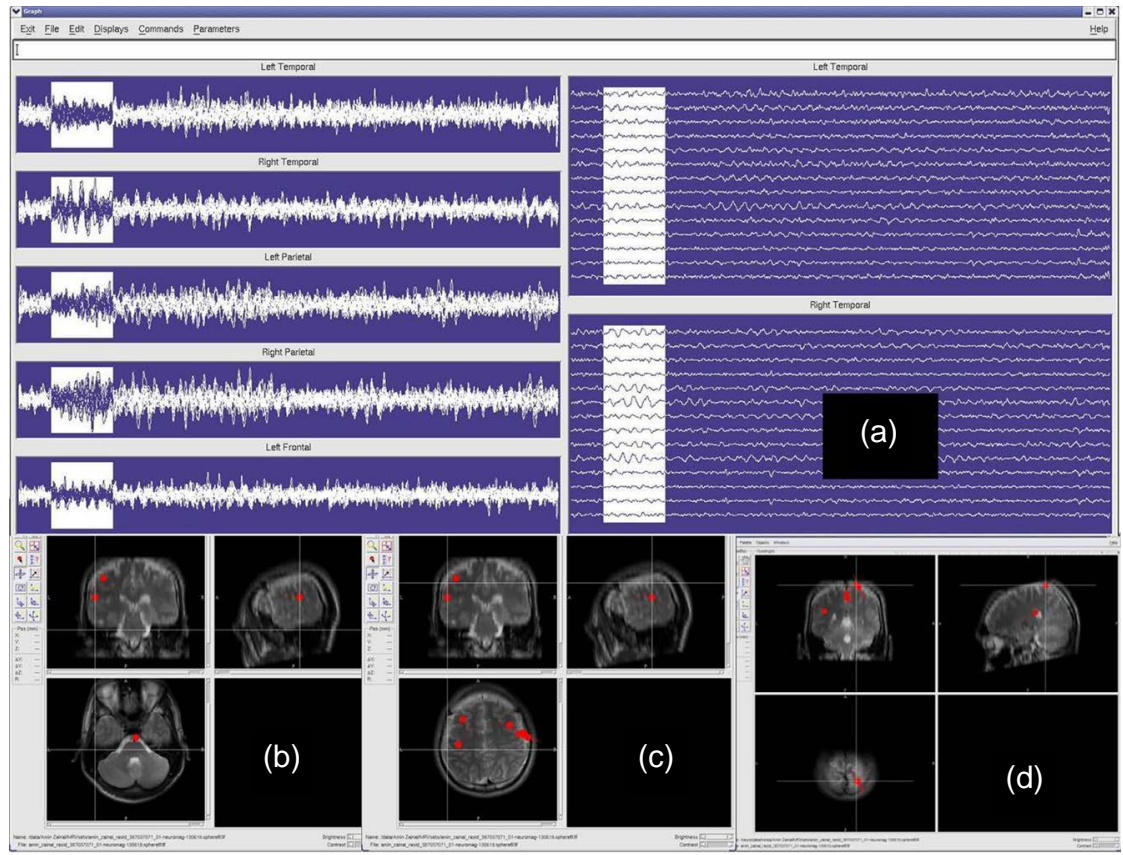

Figure 5. (a) The abnormal brainwaves for a patient suffering from complex regional pain syndrome. (b), (c) and (d) Collection of events analysis disclosed sensorimotor, insular, cingulate and amygdala-piriform-hippocampal areas are the networks for the neuropathic pain. 


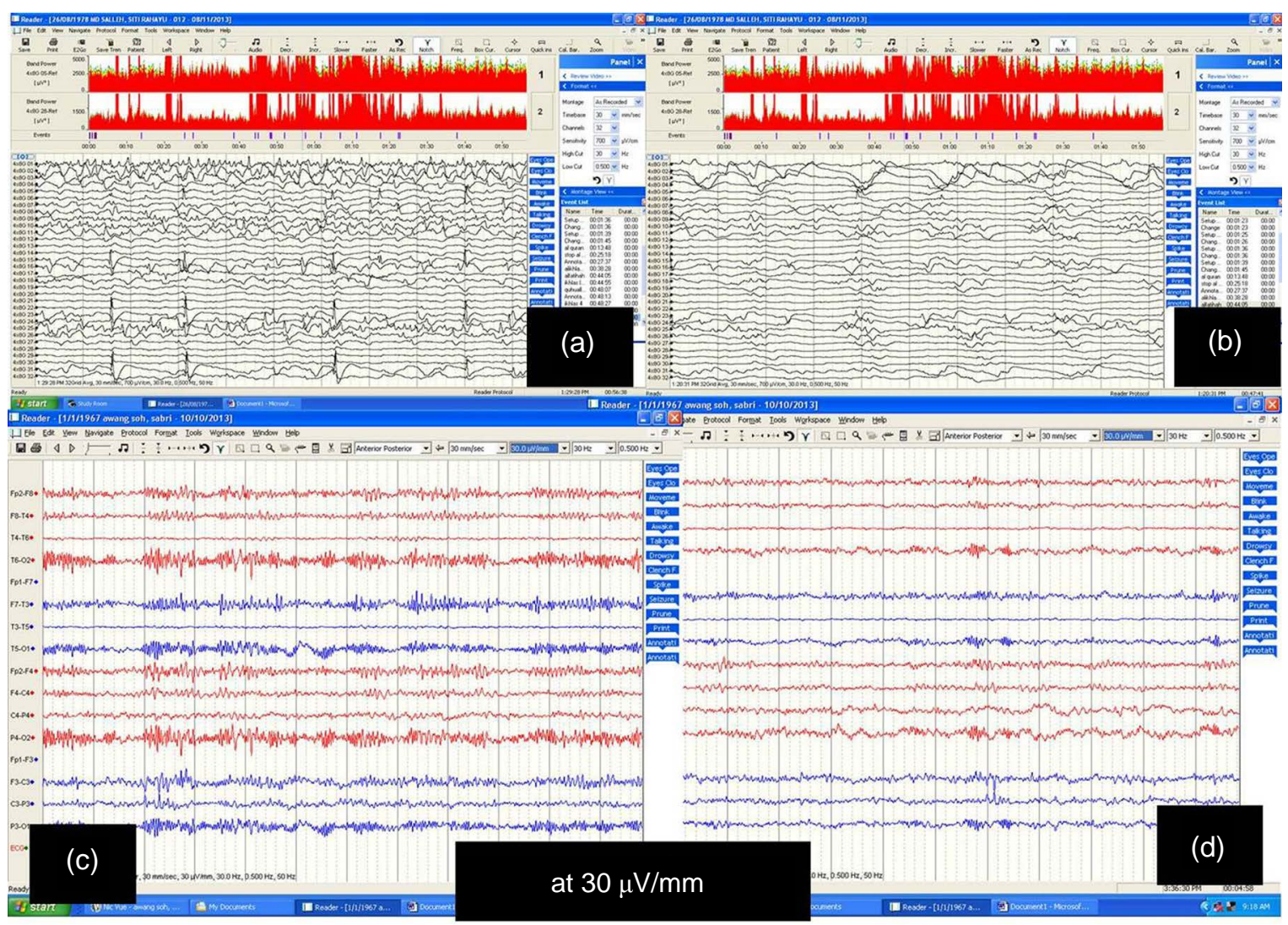

Figure 6. (a) Before and during (b) listening to religious text of the patient's faith who suffers from focal epilepsy arising at sensorimotor cortex. (c) Before and during (d) listening to the religious text of the patient's faith who suffers from convexity meningiomas at left auditory area.

Miss Alwani Liyana Ahmad and MATLAB-team.

\section{References}

[1] Zamzuri, I., Nazaruddin, W.H., Muzaimi, M., Badrisyah, I., Rahman, I.G. and Jafri, M.A. (2013) Functional MRI, Diffusion Tensor Imaging, Magnetic Source Imaging and Intraoperative Neuromonitoring Guided Brain Tumor Resection in Awake and Under General Anaesthesia. In: Lichtor, D.T., Ed., Clinical Management and Evolving Novel Therapeutic Strategies for Patients with Brain Tumors, InTech, Morn Hill, 17-54. http://dx.doi.org/10.5772/52032

[2] Ahmadlou, M., Adeli, H. and Adeli, A. (2012) Graph Theoretical Analysis of Organization of Functional Brain Networks in ADHD. Clinical EEG and Neuroscience, 43, 5-13. http://dx.doi.org/10.1177/1550059411428555

[3] Battaglia, D., Witt, A., Wolf, F. and Geisel, T. (2012) Dynamic Effective Connectivity of Inter-Areal Brain Circuits. PLOS Computational Biology, 8, Article ID: e1002438. http://dx.doi.org/10.1371/journal.pcbi.1002438

[4] Sporns, O., Tononi, G. and Edelman, G.M. (2000) Theoretical Neuroanatomy: Relating Anatomical and Functional Connectivity in Graphs and Cortical Connection Matrices. Cerebral Cortex, 10, 127-141. http://dx.doi.org/10.1093/cercor/10.2.127

[5] Momjian, S., Seghier, M., Seeck, M. and Michel, C.M. (2003) Mapping of the Neuronal Networks of Human Cortical Brain Functions. Advances and Technical Standards in Neurosurgery, 28, 91-142. http://dx.doi.org/10.1007/978-3-7091-0641-9_2

[6] Andreassi, J.L. (2007) Psychophysiology: Human Behaviour \& Physiological Response. 5th Edition, Lawrence Erlbaum Associates, New Jersey.

[7] Chang, B.S, Schomer, D.L. and Niedermeyer, E. (2011) Normal EEG and Sleep: Adults and Elderly. In: Niedermeyer, E., Ed., Electroencephalography: Basic Principles, Clinical Applications and Related Fields, Lippincott Williams \& Wilkins, Philadelphia, 183-214.

[8] Mihail, L.P. and Leon, D. (2013) Contributions to the Understanding of the Neural Bases of the Consciousness. In: 
Lichtor, D.T., Ed., Clinical Management and Evolving Novel Therapeutic Strategies for Patients with Brain Tumors, InTech, Morn Hill,

[9] Riviello, J.J.J.R., Nordli, Dr., J.R. and Niedermeyer, E. (2011) Normal EEG and Sleep: Infants to Adolescents. In: Niedermeyer, E., Ed., Electroencephalography: Basic Principles, Clinical Applications and Related Fields, Lippincott Williams \& Wilkins, Philadelphia, 163-182.

[10] Galambos, R., Makeig, S. and Talmachoff, P.J. (1981) A 40-Hz Auditory Potential Recorded from the Human Scalp. Proceedings of the National Academy of Sciences of the United States of America, 78, 2643-2647. http://dx.doi.org/10.1073/pnas.78.4.2643

[11] Gonzalez, S.L., Grave de Peralta, R., Thut, G., Millan Jdel, R., Morier, P. and Landis, T. (2006) Very High Frequency Oscillations (VHFO) as a Predictor of Movement Intentions. NeuroImage, 32, 170-179. http://dx.doi.org/10.1016/j.neuroimage.2006.02.041

[12] Crone, N.E., Sinai, A. and Korzeniewska, A. (2006) High-Frequency Gamma Oscillations and Human Brain Mapping With Electrocorticography. Progress in Brain Research, 159, 275-295.

[13] Filipe, S., Charvet, G., Foerster, M., Porcherot, J., Beche, J.F., Bonnet, S, et al. (2011) A Wireless Multichannel EEG Recording Platform. IEEE Engineering in Medicine \& Biology Society, 2011, 6319-6322.

[14] Dias, N.S., Carmo, J.P., Mendes, P.M. and Correia, J.H. (2012) Wireless Instrumentation System Based on Dry Electrodes for Acquiring EEG Signals. Medical Engineering \& Physics, 34, 972-981. http://dx.doi.org/10.1016/j.medengphy.2011.11.002

[15] Hou, K.C., Chang, C.W., Chiou, J.C., Huang, Y.H. and Shaw, F.Z. (2011) Wireless and Batteryless Biomedical Microsystem for Neural Recording and Epilepsy Suppression Based on Brain Focal Cooling. IET Nanobiotechnology, 5, 143-147. http://dx.doi.org/10.1049/iet-nbt.2011.0017

[16] Fernandez, I.S. and Loddenkemper, T. (2013) Electrocorticography for Seizure Foci Mapping in Epilepsy Surgery. Journal of Clinical Neurophysiology: Official Publication of the American Electroencephalographic Society, 30, 554570.

[17] Roberts, T.P., Ferrari, P., Perry, D., Rowley, H.A. and Berger, M.S. (2000) Presurgical Mapping with Magnetic Source Imaging: Comparisons with Intraoperative Findings. Brain Tumor Pathology, 17, 57-64. http://dx.doi.org/10.1007/BF02482736

[18] Schiffbauer, H., Berger, M.S., Ferrari, P., Freudenstein, D., Rowley, H.A. and Roberts, T.P. (2002) Preoperative Magnetic Source Imaging for Brain Tumor Surgery: A Quantitative Comparison with Intraoperative Sensory and Motor Mapping. Journal of Neurosurgery, 97, 1333-1342. http://dx.doi.org/10.3171/jns.2002.97.6.1333

[19] Alberstone, C.D., Skirboll, S.L., Benzel, E.C., Sanders, J.A., Hart, B.L., Baldwin, N.G., et al. (2000) Magnetic Source Imaging and Brain Surgery: Presurgical and Intraoperative Planning in 26 Patients. Journal of Neurosurgery, 92, 79-90. http://dx.doi.org/10.3171/jns.2000.92.1.0079

[20] Magoun, H.W. (1952) An Ascending Reticular Activating System in the Brain Stem. AMA Archives of Neurology and Psychiatry, 67, 145-154; Discussion 67-71.

[21] Steriade, M. (1996) Arousal: Revisiting the Reticular Activating System. Science, 272, 225-226.

[22] Amzica, F. and Lopes da Silva, F.H. (2011) Cellular Substrates of Brain Rhythms. In: Niedermeyer, E., Ed., Electroencephalography: Basic Principles, Clinical Applications and Related Fields, 6th Edition, Lippincott Williams \& Wilkins, Philadelphia, 33-63.

[23] Speckmann, E., Elger, C.E. and Gorji, A. (2011) Neurophysiologic Basic of EEG and DC Potentials. In: Niedermeyer, E., Ed., Electroencephalography: Basic Principles, Clinical Applications and Related Fields, Lippincott Williams \& Wilkins, Philadelphia, 17-32.

[24] Nieuwenhuys, R., Voogd, J. and van Huijzen, C. (2008) Greater Limbic System. 4th Edition, Springer-Verlag Berlin Heidelberg, Wurzburg.

[25] Nieuwenhuys, R., Veening, J.G. and van Domburg, P. (1988) Core and Paracores; Some New Chemoarchitectural Entities in the Mammalian Neuraxis. Acta Morphologica Neerlando-Scandinavica, 26, 131-163.

[26] Connor Jr., D.E., Nixon, M., Nanda, A. and Guthikonda, B. (2012) Vagal Nerve Stimulation for the Treatment of Medically Refractory Epilepsy: A Review of the Current Literature. Neurosurgical Focus, 32, E12.

[27] Schaible, H.G. (2007) Peripheral and Central Mechanisms of Pain Generation. Handbook of Experimental Pharmacology, 177, 3-28.

[28] Peyron, R., Laurent, B. and Garcia-Larrea, L. (2000) Functional Imaging of Brain Responses to Pain. A Review and Meta-Analysis (2000). Neurophysiologie Clinique=Clinical Neurophysiology, 30, 263-288.

[29] Peyron, R. and Faillenot, I. (2011) Functional Brain Mapping of Pain Perception. Medecine Sciences: M/S, 27, 82-87.

[30] Peyron, R., Garcia-Larrea, L., Gregoire, M.C., Costes, N., Convers, P., Lavenne, F., et al. (1999) Haemodynamic Brain 
Responses to Acute Pain in Humans: Sensory and Attentional Networks. Brain: A Journal of Neurology, 122, 17651780.

[31] Santoro, G., Wood, M.D., Merlo, L., Anastasi, G.P., Tomasello, F. and Germano, A. (2009) The Anatomic Location of the Soul from the Heart, through the Brain, to the Whole Body, and Beyond: A Journey through Western History, Science, and Philosophy. Neurosurgery, 65, 633-643; Discussion 643.

[32] Dolan, B. (2007) Soul Searching: A Brief History of the Mind/Body Debate in the Neurosciences. Neurosurgical Focus, 23, E2. 